\title{
THE USAGE OF INTRAVENOUS CYCLOPHOSPHAMIDE IN JUVENILE ONSET LUPUS AND ITS GESTACIONAL RESULTS IN ADULTHOOD
}

Fernanda Vieira Leão (Universidade Federal do Rio de Janeiro, Rio de Janeiro, RJ, Brasil), Raphael Neves dos Santos Mosqueira Gomes (Universidade Federal do Rio de Janeiro, Rio de Janeiro, RJ, Brasil), Blanca Elena Rios Gomes Bica (Universidade Federal do Rio de Janeiro, Rio de Janeiro, RJ, Brasil)

\section{BACKGROUND}

Cyclophosphamide (CYC) is an antineoplastic drug, introduced in the market as a chemotherapeutic agent, which, posteriorly, started being used as treatment of auto-immune diseases. For many years, it was the choice therapy, associated with glucocorticoids for the management of systemic lupus erythematosus (SLE) with renal and central nervous system involvement. Today, we give other drugs preference, such as mycophenolate and azathioprine as maintenance treatment, because of cyclophosphamide's toxicity. It is important to notice that the toxicity is cumulative dose related. As a side effect, the literature names the gonadal toxicity, which can culminate in infertility and Premature Ovarian Failure (POF).

\section{MATERIALS AND METHODS}

The medical records of 14 female patients, who have an established diagnosis of SLE which are or have been in clinical follow-up at Clementino Fraga Filho University Hospital, were evaluated and the following information was placed in worksheets: age, disease duration, number of diagnostic criteria, indication of CYC, the administration protocol (NIH or Eurotrial), time of usage, side effects, number of gestations after the usage of the medication, labours and abortions, the presence of the POF diagnose, the last registrated Systemic Lupus Erythematous Disease Activity Index (SLEDAI) and if the patient is still on follow up.

\section{RESULTS}

14 patients with a mean age of 30.8 years, a mean age of disease start of 14.1 years, and an average of 16.6 years of disease were treated with cyclophosphamide. The mean SLEDAl obtained during the last clinical evaluation was 5,2 .

$\mathrm{NIH}$ protocol was used with 11 patients, while Eurolupus protocol was used with only 3 . The motivation for the usage of $\mathrm{CYC}$ was active Nephritis in 13 patients and Pulmonary Arterial Hypertension in 1 patient. The cumulative dose varied between $1,5 \mathrm{~g}$ to $29 \mathrm{~g}$, with a mean cumulative dose of $10,83 \mathrm{~g}$. Out of the 14 patients, 8 got pregnant, giving birth to 11 newborns and 2 miscarriages. Out of the 6 patients with no offspring, 2 were still virgins, 2 used a contraceptive method and 2 didn't offer specific information regarding pregnancy prevention. No medical record displayed any report of difficulty getting pregnant and none of the patients had POF diagnosed by gynecological evaluation.

\section{CONCLUSION}

Despite the relatively high doses of CYC, the patients did not present signs of POF, neither were found clear references to infertility. 